\title{
Late-onset limb-girdle muscular dystrophy caused by GMPPB mutations
}

\author{
Balcin, Hasan
}

2017-07

Balcin , H , Palmio , J , Penttila , S , Nennesmo , I , Lindfors , M , Solders , G \& Udd , B 2017

, ' Late-onset limb-girdle muscular dystrophy caused by GMPPB mutations ' , Neuromuscular Disorders , vol. 27 , no. 7 , pp. 627-630 . https://doi.org/10.1016/j.nmd.2017.04.006

http://hdl.handle.net/10138/297794

https://doi.org/10.1016/j.nmd.2017.04.006

publishedVersion

Downloaded from Helda, University of Helsinki institutional repository.

This is an electronic reprint of the original article.

This reprint may differ from the original in pagination and typographic detail.

Please cite the original version. 
Case report

\title{
Late-onset limb-girdle muscular dystrophy caused by GMPPB mutations
}

\author{
Hasan Balcin ${ }^{\mathrm{a}, *}$, Johanna Palmio ${ }^{\mathrm{b}}$, Sini Penttilä ${ }^{\mathrm{b}}$, Inger Nennesmo ${ }^{\mathrm{c}}$, Mikaela Lindfors ${ }^{\mathrm{b}}$, \\ Göran Solders ${ }^{\mathrm{a}}$, Bjarne Udd ${ }^{\mathrm{b}, \mathrm{d}, \mathrm{e}}$ \\ ${ }^{a}$ Department of Clinical Neurophysiology and Neurology, Karolinska University Hospital, Department of Clinical Neuroscience, Karolinska Institutet, \\ Stockholm, Sweden \\ ${ }^{\mathrm{b}}$ Neuromuscular Research Center, Tampere University Hospital and University of Tampere, Finland \\ ${ }^{c}$ Department of Pathology, Karolinska Institutet and Karolinska University Hospital, Stockholm, Sweden \\ ${ }^{\mathrm{d}}$ Folkhälsan Institute of Genetics and the Department of Medical Genetics, Haartman Institute, University of Helsinki, Helsinki, Finland \\ ${ }^{\mathrm{e}}$ Department of Neurology, Vaasa Central Hospital, Vaasa, Finland
}

Received 24 November 2016; received in revised form 26 February 2017; accepted 13 April 2017

\begin{abstract}
Mutations in GMPPB gene have been reported in patients with early-onset disease ranging from severe congenital muscular dystrophies to limb-girdle muscular dystrophy (LGMD) with mental retardation. More recently mutations in GMPPB have been identified with congenital myasthenic syndromes as well as milder phenotypes. We report two unrelated cases with LGMD that underwent clinical, histopathological and genetic studies. In both cases, we found identical compound heterozygous GMPPB mutations c.79G>C p.D27H and c.859C $>$ T p.R287W, leading to a glycosylation defect of alpha-dystroglycan. The onset of muscle weakness was 30-40 years and the progression rate mild to moderate. Case 2 became wheelchair-bound at the age of 60 . No cognitive or behavioral symptoms were noted. These cases provide further evidence that $G M P P B$ mutations can also cause late-onset recessive LGMD with milder phenotypes than previously reported, and thus should be considered in the differential diagnosis of patients with adult-onset muscular dystrophies.
\end{abstract}

(C) 2017 Elsevier B.V. All rights reserved.

Keywords: Limb-girdle muscular dystrophy; Alpha-dystroglycan; GMPPB gene; Late-onset

\section{Introduction}

Dystroglycanopathy is a collective term for a heterogeneous group of muscular dystrophies caused by aberrant glycosylation of alpha-dystroglycan ( $\alpha$-DG), a sarcolemmal structural membrane protein, and leading to a loss of its laminin-binding activity [1-3]. Dystroglycanopathies have a wide clinical spectrum from severe forms of congenital muscular dystrophies (CMD) with brain and eye-abnormalities, to milder phenotypes such as limb-girdle muscular dystrophy type 2I (LGMD2I). To date, mutations in 18 genes have been associated with glycosylation defect of $\alpha-\mathrm{DG}$ and all of them but DAG1-related mutations are known to cause secondary dystroglycanopathies [4,5].

$G M P P B$ gene encoding guanosine diphosphate mannose pyrophosphorylase $\mathrm{B}$ protein plays an important role in the

\footnotetext{
* Corresponding author. Department of Clinical Neurophysiology (C1-78), Karolinska University Hospital (Huddinge Site) Hälsovägen 1, 14186 Stockholm, Sweden. Fax: +4685 8582030.

E-mail address: hasan.balcin@gmail.com (H. Balcin).
}

glycosylation of proteins and lipids [6], including alphadystroglycan. Mutations in $G M P P B$ cause hypoglycosylated $\alpha-D G$ with phenotypes ranging from CMD with brain and eye abnormalities to severe intellectual disability and epilepsy to a case resembling LGMD with only mild cognitive disability [4,7-9]. The first reported cases had an early childhood-onset and typically severe disease with central nervous system (CNS) involvement. Later GMPPB-mutated adult- or adolescenceonset LGMD with or without intellectual disability was described [10-12]. More recently congenital myasthenic syndromes have been associated with GMPPB mutations as well $[13,14]$. In this report, we present two additional unrelated patients with milder adult-onset LGMD without CNS involvement caused by GMPPB mutations.

\section{Case reports}

\subsection{Case 1}

A 40-year-old woman of Swedish-Belgian descent, born to non-consanguineous parents, with normal motor milestones 


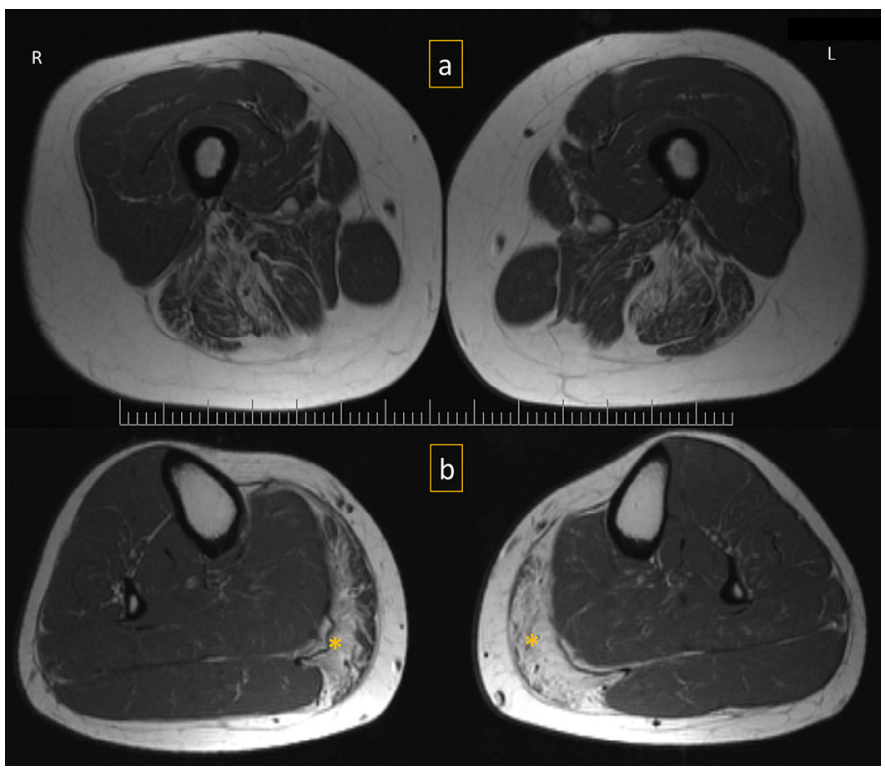

Fig. 1. Muscle imaging findings. Muscle MRI (Case 1), axial T1-weighted images, 1.5 Tesla, illustrating lower limbs, showing fat and connective tissue replacement in the posterior compartment of the thigh (a), especially of the hamstring muscles and of the medial gastrocnemius $\left(^{*}\right)$ muscle bilaterally in the lower leg (b)

and without prior medical history. At the age of 15 , the patient noticed poor performance in school sports without obvious weakness or exercise intolerance. Due to persistent elevation of liver enzymes at the age of 17 , she underwent liver biopsy with a diagnosis of unspecified hepatitis. Aged 20, she had trouble climbing stairs and rising from the floor. On clinical examination, aged 37 , she had waddling gait with a prominent Trendelenburg sign on the left side, left-sided scapular winging, mild calf hypertrophy, increased lumbar lordosis, and shortness of breath in the supine position without signs of nocturnal hypoventilation. Muscle testing (MRC) revealed: elbow flexion/extension and shoulder abduction 4/5, knee extension/ flexion $3+/ 5$, hip flexion/extension and abduction $2 / 5$, neckextension $4 / 5$, neck-flexion $3+/ 5$. Distal muscle strength was normal. CK values were 3-5 times of the upper limit of normal values (ULN) and liver enzymes, alanine aminotransferase and aspartate aminotransferase, varied between 1.5 and 5 times of ULN during the last decade. Echocardiography and $24 \mathrm{~h}-$ Holter-EKG were normal. Spirometry on two occasions showed slightly reduced forced vital capacity (78-84\% predicted). EMG showed a myopathic pattern in the proximal muscles without spontaneous activity. By the age of 39, muscle weakness had progressed to involve the neck muscles, and the patient uses calipers indoor and electric scooter outdoor. Muscle MRI showed fatty degenerative changes in lumbar paravertebral, gluteus minimus, adductor longus, medial gastrocnemius and less pronounced in gluteus medius, semitendinosus, biceps femoris long head and adductor magnus muscles, without presence of notable edema (Fig. 1). No cognitive, behavioral, sensory, bulbar or cardiac symptoms have been reported by the patient. Brain MRI was normal.

\subsection{Case 2}

A 69-year-old Finnish woman, born to non-consanguineous parents, had unremarkable childhood and adolescence, and negative family history. She noticed first signs of muscle symptoms in her 30s, and progressive muscle weakness after the age of 40 with difficulties in climbing stairs, lifting objects and in ordinary walking but continued working until age 53. On initial examination, proximal weakness was evident and more pronounced in the lower limbs, gait was waddling but distal muscle strength preserved. The calves were pseudohypertrophic. The progression of the disease was moderate but steady; she started to use sticks at age 53 and motorized/electrical wheelchair at age 60. At the last examination (age 67) there was no active movement in the pelvic muscles or in ankle dorsal and plantar flexion. Knee extension and flexion was 2-2.5/5. Elbow and finger extension and flexion was $4 / 5$, but arm abduction less than 90 degrees. There was no facial, bulbar or neck muscle weakness, no contractures or scapular winging. EMG showed myopathic changes and widespread spontaneous activity. CK values were constantly elevated up to 10 times ULN and liver enzyme alanine aminotransferase was elevated twofold. Echocardiogram and spirometry tests showed normal results. Cognitive or behavioral disturbances have not been noticed.

\subsection{Muscle biopsy findings}

\subsubsection{Case 1}

Biopsy from the deltoid muscle and vastus lateralis showed fiber-size variation, and fibers containing increased amount of internalized nuclei in both muscles. Necrotic fibers were present in the deltoid muscle (Fig. 2A). Immunohistochemical evaluation of dystrophin (DYS 1-3), beta-dystroglycan, spectrin, alpha-, beta-, gamma- and delta-sarcoglycan, myotilin (all from Novocastra NCL), merosin (Chemicon) and caveolin-3 (Santa Cruz Biotechnology) showed normal expression. Additional immunohistochemistry of $\alpha-D G$ was performed on four occasions and each time revealed irregular patchy expression (Fig. 2B, C). Western blot analysis (WB) was conducted for calpain-3 and dysferlin (both from Novocastra NCL) with normal results. WB for $\alpha-D G$ (Santa Cruz Biotechnology, (IIH6)) showed a markedly reduced immunostaining (Fig. 2D). The finding indicated a glycosylation defect of $\alpha-\mathrm{DG}$.

\subsubsection{Case 2}

Three muscle biopsies from both distal and proximal muscles indicated general dystrophic changes: atrophic fibers, necrosis, fibrosis, and excess of fat. Immunohistochemistry performed 20 years earlier using DYS 2 antibody (Novocastra NCL) showed uneven staining and also WB showed reduced amount of DYS 2 leading to initial diagnosis of manifesting female carrier of dystrophinopathy.

\subsection{Molecular genetic studies}

Initial genetic analyses, such as FKRP, CAPN3, DMD and $D Y S F$, showed normal results. Targeted next generation sequencing was performed as previously described [15] using version 2 of the MYOcap gene panel that is targeted to the exons 


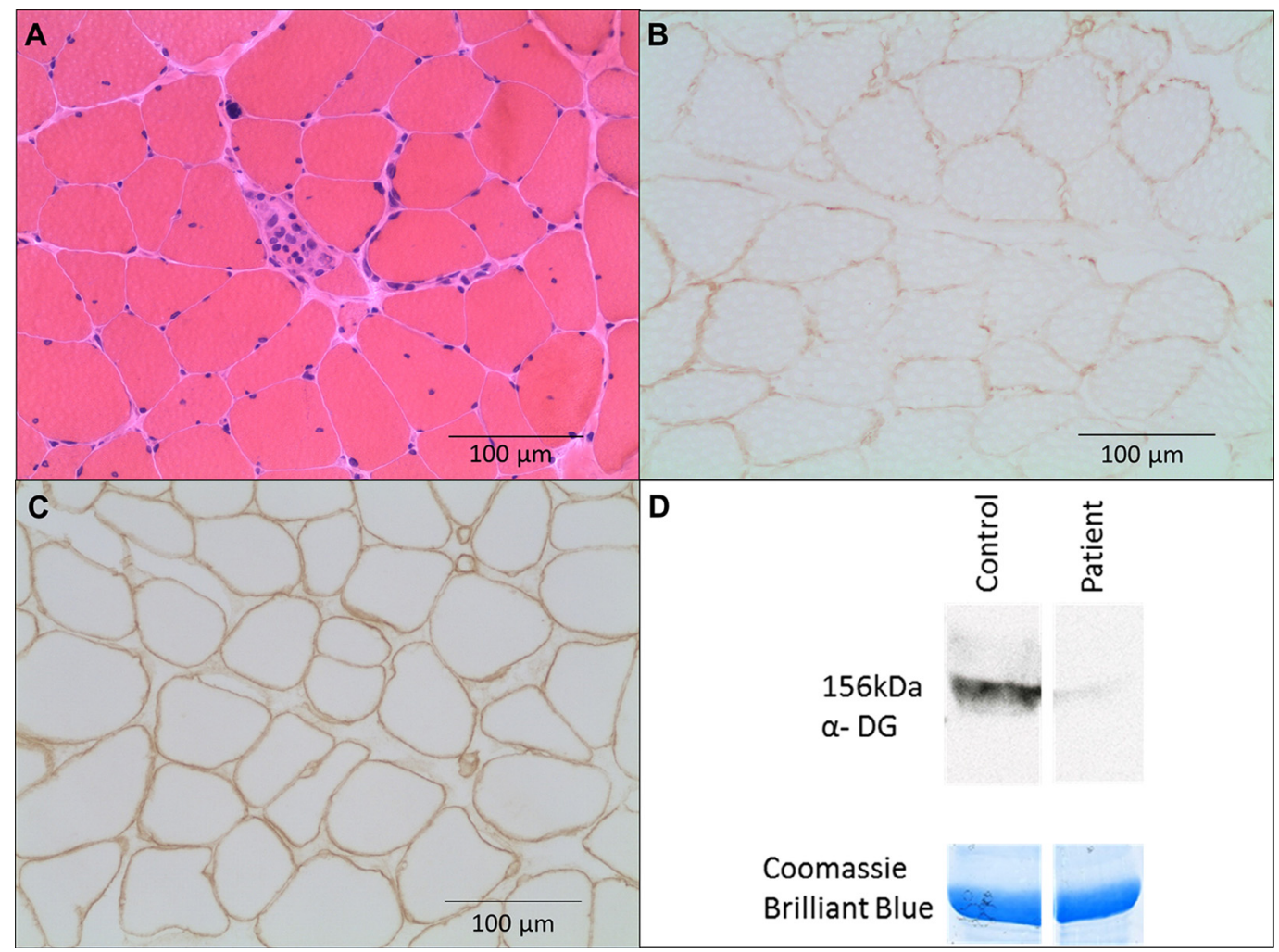

Fig. 2. Muscle biopsy findings. H\&E-staining showing dystrophic patterns of deltoid muscle from Case 1 with fiber size-variation, and one necrotic muscle fiber (A). Immunohistochemistry of a-dystroglycan ( $\alpha$-DG, Santa Cruz Biotechnology, (IIH6)) revealed irregular patchy expression (B). Normal expression of $\beta$-DG (Novocastra NCL) demonstrates intact sarcolemma (C). Western blot analysis of $\alpha$-DG shows normal expression of staining for $\alpha$-DG in muscle samples from healthy controls, while markedly reduced in Case 1 (D). Coomassie staining demonstrating similar loading of skeletal muscle proteins across samples. Muscle samples from healthy individuals were used as positive control (C50). Protein quantity was standardized with myosin.

of 236 genes known or predicted to cause muscular dystrophy or myopathy. A more precise description of the MYOcap gene panel and data analysis is given in Supplementary File S1.

The analysis of the patient samples revealed compound heterozygosity of two identical mutations in GMPPB in both patients: mutations c. $79 \mathrm{G}>\mathrm{C}$ p.D27H and c.859C $>$ T p.R287W both previously reported $[4,7,10-13]$. The mutations were verified by Sanger sequencing.

\section{Discussion}

$G M P P B$ mutations were first identified in eight different unrelated pediatric patients with severe very early-onset muscular dystrophies, typically with CNS involvement [7]. Together with few recent reports [10-12], we can now confirm milder adult-onset LGMD without CNS involvement caused by compound heterozygous mutations in GMPPB. As with other dystroglycanopathies the primary mutations lead to a glycosylation defect of $\alpha$-DG.

Interestingly, we identified in both totally unrelated cases the identical compound heterozygous mutations. The c.79G>C p.D27H mutation seems to be a common variant and it has been associated with early-onset LGMD with or without epilepsy and mental retardation $[4,7]$, although more commonly with milder and late-onset LGMD [10-12]. The second c.859C $>\mathrm{T}$ p.R287W mutation has been previously identified in only five patients. One of them had CMD with cerebellar involvement [11]. Four patients had the same mutations in compound heterozygosity as our patients; one with the disease classified as congenital myasthenic syndrome [13] and three with LGMD phenotype and no CNS dysfunction, disease-onset ranging from five to 30 years [12]. Two of the latter patients underwent muscle imaging that showed severe fatty degeneration in paraspinal muscles, hamstring muscles and to a lesser degree in calf muscles [12]. The affected muscles in our patient showed the same distribution, although our patients are at the milder end of the disease spectrum.

Cabrera-Serrano et al. [10] reported eight patients with lateonset LGMD among five different families. Most of those patients had some degree of cognitive and/or behavioral symptoms or signs except patients 3 and 4 in family II, who were clinically most similar to our cases and shared the same pathogenic heteroallelic mutation c.79G>C. Similarly, in the report of Carss et al. [7] the patient with the mildest phenotype among all eight early-onset CMD/LGMD cases and the only one without CNS signs carried the mutation c.79G $>$ C on one allele. In the transfection studies with the different mutations, again this c.79G $>\mathrm{C}$ showed less abnormality than the ones leading to severe CMD-CNS phenotypes [7]. These observations clearly suggest that patients with the mutation c. $79 \mathrm{G}>\mathrm{C}$ have a milder course of the disease because of less severe and only partial glycosylation defect of $\alpha$-DG observed on blots and as irregular 
patchy immunostaining label for $\alpha-D G$, as shown in Case 1 and reported by others. However, there might also be other genetic or epigenetic factors mediating the partial glycosylation defect of $\alpha$-DG.

The presented cases provide further evidence for the occurrence of a much milder recessive LGMD phenotype caused by $G M P P B$ mutations, in particular associated with the mutation c.79G $>$ C. Finally, we suggest that $G M P P B$-related LGMD should be considered in the differential diagnosis of patients with late-onset muscular dystrophy, especially in cases with pathological immunostaining for $\alpha-\mathrm{DG}$ and normal FKRP gene analysis.

\section{Acknowledgements}

The authors thank the patients for participation in this work.

\section{Appendix: Supplementary material}

Supplementary data to this article can be found online at doi:10.1016/j.nmd.2017.04.006.

\section{References}

[1] Barresi R, Campbell KP. Dystroglycan: from biosynthesis to pathogenesis of human disease. J Cell Sci 2006;119:199-207.

[2] Ibraghimov-Beskrovnaya O, Ervasti JM, Leveille CJ, Slaughter CA, Sernett SW, Campbell KP. Primary structure of dystrophin-associated glycoproteins linking dystrophin to the extracellular matrix. Nature 1992;355:696-702.

[3] Muntoni F, Torelli S, Brockington M. Muscular dystrophies due to glycosylation defects. Neurother 2008;5:627-32.

[4] Bharucha-Goebel DX, Neil E, Donkervoort S, Dastgir J, Wiggs E, Winder TL, et al. Intrafamilial variability in GMPPB-associated dystroglycanopathy: broadening of the phenotype. Neurology 2015;84: 1495-7.

[5] Bönnemann CG, Wang CH, Quijano-Roy S, Deconinck N, Bertini E, Ferreiro A, et al. Diagnostic approach to the congenital muscular dystrophies. Neuromuscul Disord 2014;24:289-311.

[6] Maeda Y, Kinoshita T. Dolichol-phosphate mannose synthase: structure, function and regulation. Biochim Biophys Acta 2008;1780:861-8.

[7] Carss KJ, Stevens E, Foley AR, Cirak S, Riemersma M, Torelli S, et al. Mutations in GDP-mannose pyrophosphorylase B cause congenital and limb-girdle muscular dystrophies associated with hypoglycosylation of alpha-dystroglycan. Am J Hum Genet 2013;93:29-41.

[8] Raphael AR, Couthouis J, Sakamuri S, Siskind C, Vogel H, Day JW, et al. Congenital muscular dystrophy and generalized epilepsy caused by GMPPB mutations. Brain Res 2014;1575:66-71.

[9] O'Grady GL, Lek M, Lamande SR, Waddell L, Oates EC, Punetha J, et al. Diagnosis and etiology of congenital muscular dystrophy: we are halfway there. Ann Neurol 2016;80:101-11.

[10] Cabrera-Serrano M, Ghaoui R, Ravenscroft G, Johnsen RD, Davis MR, Corbett A, et al. Expanding the phenotype of GMPPB mutations. Brain 2015;138:836-44.

[11] Jensen BS, Willer T, Saade DN, Cox MO, Mozaffar T, Scavina M, et al. GMPPB-associated dystroglycanopathy: emerging common variants with phenotype correlation. Hum Mutat 2015;36:1159-63.

[12] Oestergaard ST, Stojkovic T, Dahlqvist JR, Bouchet-Seraphin C, Nectoux $\mathrm{J}$, Leturcq F, et al. Muscle involvement in limb-girdle muscular dystrophy with GMPPB deficiency (LGMD2T). Neurol Genet 2016;2:e112.

[13] Belaya K, Rodríguez Cruz PM, Liu WW, Maxwell S, McGowan S, Farrugia ME, et al. Mutations in GMPPB cause congenital myasthenic syndrome and bridge myasthenic disorders with dystroglycanopathies. Brain 2015;138:2493-504.

[14] Rodríguez Cruz PM, Belaya K, Basiri K, Sedghi M, Farrugia ME, Holton $\mathrm{JL}$, et al. Clinical features of the myasthenic syndrome arising from mutations in GMPPB. J Neurol Neurosurg Psychiatry 2016;87:802-9.

[15] Evilä A, Arumilli M, Udd B, Hackman P. Targeted next-generation sequencing assay for detection of mutations in primary myopathies. Neuromuscul Disord 2016;26:7-15. 